\title{
USO DE BASTÓN Y CAÍDAS EN ADULTOS MAYORES CHILENOS DE LA COMUNIDAD ${ }^{1}$
}

\section{CANE USE AND FALLS IN CHILEAN COMMUNITY-DWELLING OLDER ADULTS}

\author{
José Alex Leiva-Caro* \\ José MANuel León-Pino ** \\ Juana Elena Cortés-Recabal ${ }^{* * *}$
}

\begin{abstract}
RESUMEN
Objetivo: Describir las características sobre el uso del bastón y la prevalencia de caídas en adultos mayores de la comunidad que han recibido ayuda técnica en un Centro de Salud Familiar. Material y método: Estudio cuantitativo, descriptivo y transversal. La muestra correspondió a 64 adultos mayores de entre 60 y 94 años. Para recolectar los datos se utilizó un cuestionario que evaluó variables sociodemográficas, de salud, uso de bastón y caídas. El análisis estadístico se realizó mediante el paquete SPSS. Se utilizó consentimiento informado, considerando los lineamientos sobre investigación y confidencialidad de la información dispuestos en Chile. Resultados: El 95,3\% refirió saber el motivo de uso del bastón; el 7,8\% utiliza el bastón cuando se moviliza dentro de la casa, el $45,3 \%$ cuando se moviliza fuera de la casa, el 46,9\% cuando se moviliza por todas partes; y el $48,4 \%$ expresó que no le agrada utilizar el bastón. La prevalencia de caídas en los últimos seis meses fue de $73 \%$; de quienes presentaron caídas el 61\% no estaba usando el bastón al momento de caer, y el 94\% expresó tener miedo de sufrir una caída. Conclusión: Los resultados muestran que un alto porcentaje de adultos mayores no está cumpliendo con el uso del bastón, por lo tanto el objetivo de su entrega no se está logrando. Se debe incluir la evaluación y educación del uso del bastón como una actividad que forme parte del control de salud del adulto mayor.
\end{abstract}

Palabras clave: Bastones, caídas, adulto mayor, enfermería geriátrica.

\begin{abstract}
Aim: To describe the characteristics of cane use and prevalence of falls in community-dwelling older adults who have received technical assistance in a Family Health Center. Method: Quantitative, descriptive and crosssectional study. Sample consisted of 64 older adults between 60 and 94 years of age. A questionnaire addressing socio-demographics, health, cane use and fall-related information was used to collect the data. Statistical analysis was performed with SPSS. An informed consent form was delivered and designed in compliance with Chile's guidelines on research and confidentiality of information. Results: $95.3 \%$ of participants reported knowing the motives for cane use; $7.8 \%$ use a cane while walking within home; $45.3 \%$ use a cane while walking outdoors;

\footnotetext{
${ }^{1}$ Proyecto de Investigación UBB-FDI 14-06. Universidad del Bío-Bío.

* Doctor en Ciencias de Enfermería, Grupo de Investigación en Envejecimiento, Departamento de Enfermería, Facultad Ciencias de la Salud y de los Alimentos, Universidad del Bío-Bío, Chillán, Chile. Email: jleiva@ubiobio.cl

${ }^{* *}$ Estudiante Licenciatura en Enfermería, Auxiliar de Investigación Grupo de Investigación en Envejecimiento, Escuela de Enfermería Universidad del Bío-Bío, Chillán, Chile. Email: josleon@alumnos.ubiobio.cl

${ }^{* * *}$ Magíster en Familia, Departamento de Enfermería, Facultad Ciencias de la Salud y de los Alimentos, Universidad del Bío-Bío, Chillán, Chile. Email: jcortes@ubiobio.cl
} 
$46.9 \%$ use a cane to walk anywhere; and $48.4 \%$ said that they do not like to use the cane. The prevalence of falls in the last six months was $73 \% ; 61 \%$ of those who have fallen were not using a cane when they fell, and $94 \%$ expressed fear of falling. Conclusion: The findings show that a high percentage of older adults is not using a cane, and therefore the ultimate goal of delivering mobility devices is not being achieved. Cane use evaluation and education should be considered as an older adults' health control measure.

Key words: Canes, accidental falls, aged, geriatric nursing.

Fecha recepción: 13/06/16 Fecha aceptación: 15/11/17

\section{INTRODUCCIÓN}

En los Estados Unidos 8,5 millones de adultos mayores (AM) han reportado utilizar algún dispositivo de ayuda para caminar, siendo el bastón el más utilizado (1), situación que se repite en Chile, constituyéndose en la ayuda técnica más utilizada por los AM, seguida por el andador. Se considera que el aumento de utilización de ayudas técnicas es directamente proporcional al incremento de la edad; por lo tanto, al acrecentar la expectativa de vida y por ende el número de AM se prevé que la utilización de ayudas técnicas aumente, entre ellas el bastón (2).

El bastón es un elemento que puede ser utilizado en forma transitoria o permanente, de uso transversal a los grupos de edad y sexo. En Chile, en forma transitoria se indica a personas de 65 años y más como parte del programa de rehabilitación posterior a endoprótesis total de cadera (3) y en forma permanente a quienes presenten secuelas de accidente cerebrovascular, amputaciones de extremidades inferiores, entre otras indicaciones (4). A nivel nacional, además se cuenta con programas que garantizan la entrega de ayudas técnicas a la población de AM (5), siendo controlado el uso de la ayuda técnica al siguiente mes de su entrega, según la Guía Clínica, por el profesional responsable de las ayudas técnicas en el centro de salud respectivo, estableciéndose que el equipo de salud será el encargado del seguimiento posterior
(4). En este contexto, cobra relevancia el profesional de enfermería, pues es quien realiza controles periódicos al AM y visitas al domicilio, donde se constata el uso de la ayuda técnica en el entorno habitual y factores de riesgo de caídas.

Sin embargo, si bien el bastón es reconocido como algo necesario por los AM, también es un recordatorio constante de sus limitaciones y su utilización implica en algunos casos transacciones complejas entre el componente personal, medio ambiente y la actividad a desarrollar (6). Es decir, un AM que utiliza bastón, que además tiene disminución de la agudeza visual y que debe enfrentar entornos con desniveles en el piso, baja o nula luminosidad, sin duda se verá enfrentado a una situación compleja al interactuar con el ambiente, que lo puede llevar a presentar caídas.

Es por ello que el uso del bastón ha sido considerado un factor de riesgo de caída en el AM (7); así también se han reportado como riesgos de caer el uso de múltiples medicamentos, inestabilidad para caminar, edad avanzada, vivir solo, antecedentes de caídas y miedo a caer, entre otros (8). A su vez, las caídas producen diversas consecuencias, como lesiones leves (9), tener que utilizar bastón u otra ayuda técnica, dependencia de otras personas $(10)$, fracturas $(8,11)$ prolongado tiempo de hospitalización, cuidados a largo plazo en domicilio y muerte (12). En lo económico, las caídas en los AM son consideradas como una la lesión de elevado costo (12-14), debido a las consultas en servicios 
de urgencia, hospitalización, tratamientos y cuidados, lo que afecta a organismos de salud y familia. Por consiguiente, se ha reportado que el costo promedio de las prestaciones en salud derivado de una caída en AM es de US $\$ 10.600$ (13), cifra que se incrementa al tratar AM con fractura de cadera a consecuencia de la caída, llegando a sobrepasar los US $\$ 57.000$ (12).

En Chile la información sobre el uso de bastón y caídas en AM es exigua. Se han recuperado tres investigaciones sobre caídas, todas realizadas en la ciudad de Santiago, siendo una en AM de la comunidad (15) y otra en institucionalizados (16); el tercer estudio se realizó con datos del proyecto Salud, Bienestar y Envejecimiento en América Latina y el Caribe, que consideró una muestra de AM de la comunidad (17). Es necesario precisar que los estudios de caídas no son recientes, pues fueron publicados en los años 2001, 2003 y 2005 respectivamente. En cuanto al bastón, se recuperó un estudio (18) que se enfocó en evaluar el cumplimiento de Garantías Explícitas en Salud de la ayuda técnica en una comuna de la Región Metropolitana. Respecto a la baja cantidad de estudios recuperados sobre caídas a nivel nacional, quizá se deba a la falta de interés en estudiar el tema y distintos fenómenos de la población de AM, ya que con frecuencia las caídas no son reconocidas como un problema a menos que tengan algún efecto dañino (19), como las fracturas.

En la revisión de literatura sobre caídas y uso de bastón se manifiesta que el bastón es un factor de riesgo de caídas (7), pero no se realiza una evaluación a profundidad de las características del riesgo (ejemplo, mal uso del bastón) y, por otro lado, se recomienda el uso del bastón en las personas con riesgo de caer (4). Esto insta a desarrollar mayor investigación abordando el uso del bastón como agente causal de caídas, factor de riesgo o de consecuencias en los AM de la comunidad, entre otros temas.

Por lo expuesto, sumado a que la investi- gación sobre uso del bastón y caídas como tema central de estudio se encuentra en desarrollo en el contexto internacional y que en Chile no se ha recuperado evidencia, el propósito que se planteó en este estudio fue describir las características sobre el uso del bastón y la prevalencia de caídas en AM de la comunidad que han recibido la ayuda técnica en un Centro de Salud Familiar urbano. Los resultados pueden ser útiles para profesionales de enfermería y otros integrantes del equipo de salud, para diseñar estrategias sobre el uso del bastón y prevención de caídas en AM de la comunidad.

\section{MATERIAL Y MÉTODO}

Estudio cuantitativo, descriptivo y transversal, que se llevó a cabo en una comuna de la Provincia de Nuble, Chile. La población estuvo compuesta por AM hombres y mujeres de 60 años y más que recibieron bastón en un Centro de Salud Familiar (CESFAM) de la zona urbana desde agosto de 2013 hasta agosto de 2014. La población correspondió en total a $79 \mathrm{AM}$, siendo la muestra la totalidad de quienes recibieron bastón. Se excluyó aquellas personas que no escucharon la voz del entrevistador, el entrevistador no entendió lo que decían, debido a voz inaudible o no articulada, o que expresaron requerir apoyo de otra persona para caminar y los AM invidentes. En suma, un AM no cumplió criterios de inclusión y a 14 no fue posible incorporarlos (12 por cambio de domicilio y 2 por fallecimiento); por lo tanto la muestra quedó integrada por $64 \mathrm{AM}$.

En primera instancia se procedió a solicitar autorización por escrito a la Dirección del CESFAM para obtener antecedentes de los AM que habían recibido bastón. Luego, un auxiliar de investigación (AI) realizó llamado telefónico a cada AM para informar de la investigación: el objetivo, corroborar criterios 
de inclusión, deseo de participar y acordar una fecha de visita al domicilio. Acto seguido se procedió a realizar la recolección de datos desde octubre de 2014 a marzo de 2015, para lo cual se utilizó un cuestionario que fue aplicado por el AI. El cuestionario, denominado Uso de Bastón y Caídas en Adultos Mayores, fue creado por el autor principal, tomando como base lo realizado en una investigación previa (20), el cual fue sometido a revisión de expertos $(n=8)$ en metodología de la investigación y los fenómenos que afectan a los AM, luego se realizó una prueba piloto a $15 \mathrm{AM}$ de similares características, todos usuarios de bastón inscritos en un CESFAM de otra comuna. El cuestionario abordó variables sociodemográficas como: sexo, edad, educación, número de personas con las que vive el $\mathrm{AM}$, enfermedades crónicas, consumo diario de medicamentos. Asimismo, incluyó información sobre tiempo de uso del bastón, motivo de uso, quién le enseño a utilizarlo, lugar de uso, seguridad que percibe al utilizarlo, preferencia del uso, apreciación de cuánto camina desde que utiliza el bastón y agrado al utilizarlo. Para las caídas se preguntó, entre otros, por presencia del evento hasta seis meses antes de la evaluación, la causa de la caída, lugar de la caída y miedo a sufrir una caída. La variable miedo se clasificó como dicotómica con respuesta si y no (20). El análisis de datos se realizó en el paquete estadístico Statiscal Package for the Social Sciences (SPSS) versión 21 para Mac OSX; se utilizó estadística descriptiva como medidas de tendencia central, frecuencias y porcentajes.

Para efectos del estudio se consideraron los lineamientos de la Ley 20.120 (21) y Ley 19.628 (22) dispuestas en Chile, sobre investigación y confidencialidad de la información respectivamente, y contó con la aprobación del Comité de Bioética y Bioseguridad de la Universidad del Bío-Bío. El consentimiento informado fue aplicado en forma individual a cada AM. Posterior a su firma se procedió a la recolección de la información.

\section{RESULTADOS}

Se entrevistaron $64 \mathrm{AM}$ en su domicilio, de éstos el $62,5 \%(n=40)$ correspondió a mujeres, el rango de edad fue de 60 a 94 años, el rango con la mayor concentración de AM fue de 70 y más años $(62,5 \%, n=40)$, seguido por el rango de 65 a 70 años $(26,6 \%, n=17)$, y finalmente de 60 a 64 años con el 10,9\% (n=7). Respecto a la educación, el 14,1\% (n=9) eran analfabetos; misma cifra que manifestaron vivir solos $(14,1 \%, n=9)$. En cuanto a salud, el $100 \%(n=64)$ refirió tener alguna enfermedad crónica diagnosticada por el médico, siendo la hipertensión arterial $(84,4 \% ; n=54)$ la que se presentó en mayor porcentaje, seguida del colesterol elevado $(51,6 \% ; n=33)$ y diabetes $(48,4 \% ; n=31)$. A su vez, el $96,9 \%$ $(n=62)$ se encuentra en tratamiento farmacológico, con una media de consumo diario de 5,8 $(\mathrm{DE}=2,0)$ medicamentos. La proporción de caídas atribuidas a consumo de medicamentos -según información aportada por el AM- fue mayor en quienes consumían 4 y más medicamentos por día $(72 \%, n=34)$, llegando a un consumo máximo diario de 8 medicamentos.

Respecto al uso del bastón, fue superior a un año con un 70,3\% ( $\mathrm{n}=45)$. Al consultar por qué debía utilizar el bastón, el 95,3\% $(n=61)$ refirió saber el motivo de uso. Por su parte, el 76,6\% ( $n=49)$ aseveró que les habían educado respecto a cómo debía utilizar la ayuda técnica; ahora bien, entre los responsables de haber dado la educación el primer lugar lo ocupó el kinesiólogo $(69,4 \%$; $n=34)$, seguido por el médico $(12,2 \% ; n=6)$, la/el enfermera/o $(8,2 \% ; n=4)$ y otros, como familiares y amigos $(10,2 \%$; $n=5)$. Se preguntó por el uso del bastón en función del ambiente físico, el 7,8\% (n=5) refirió que lo utiliza solo cuando se moviliza dentro de la casa, el 45,3\% ( $n=29)$ solo cuando se moviliza fuera de la casa y el $46,9 \%(n=30)$ cuando se moviliza por todas partes. Además, se le 
preguntó al AM qué tan seguro se sentía al usar el bastón, el 89,1\% (n=57) refirió sentirse seguro, el 7,8\% $(\mathrm{n}=5)$ medianamente seguro y el 3,1\% $(n=2)$ nada seguro. Asimismo, sobre la preferencia del uso del bastón respecto a otro apoyo para caminar, el $70,3 \%(n=45)$ de los AM manifestaron que preferían el bastón como medio de apoyo y el 29,7\% $(n=19)$ preferían apoyarse en otra persona para caminar. Ahora bien, desde que tienen el bastón, el 54,7\% ( $n=35)$ manifestó que caminaba menos, seguido por el $25 \%$ $(\mathrm{n}=16)$ que refirió que caminaba lo mismo y el 20,3\% (n=13) que caminaba más. Por otro lado se preguntó sobre el agrado de utilizar el bastón, el 48,4\% $(n=31)$ expresó que no le agradaba, el 45,3\% $(n=29)$ que le agradaba y el 6,3 $(n=4)$ que le era indiferente utilizarlo.

Respecto a las caídas, el 73,4\% $(n=47)$ manifestó haber presentado caídas hasta seis meses previos a la entrevista, de éstos el $53,2 \%(n=25)$ presentó una caída y el 46,8\% $(\mathrm{n}=22)$ presentó 2 o más caídas. De quienes presentaron una o más caídas, el 85\% (n=40) vivía con una o más personas; de quienes cayeron, el 61,7\% (n=29) no estaba usando el bastón al momento de la caída.

Respecto a la ocurrencia de caídas por grupo etario, se obtuvo que la mayor prevalencia fue entre los 66-70 años, siendo la media de edad de quienes presentaron caídas 76 años $(\mathrm{DE}=8,6)$. Respecto al sexo y la presencia de caídas, se obtuvo que las mujeres se caen más que los hombres con un 63,8\% $(n=30)$. Del total de la muestra el 88,2\% $(n=60)$ expresó tener miedo de sufrir una caída, porcentaje que aumenta cuando solo se evalúa a aquellos que presentaron una o más caídas (Tabla 1). En relación al lugar de la caída, evaluado como interior y exterior de la casa, se presentaron fuera de la casa en un 55,3\% $(n=26)$ y en el interior en un 44,7\% $(n=21)$. En específico, las caídas fuera de la casa se presentaron en el patio, antejardín $=23,4 \%(n=11)$ y la calle, centro comercial, otros $=31,9 \%,(n=15)$; en cuanto a la causa de la caída, que fue re-

Tabla 1. Prevalencia de caídas en adultos mayores de la comunidad según edad, sexo y miedo de sufrir una caída $(n=64)$.

\begin{tabular}{llrrrr}
\hline Variables & & \multicolumn{3}{c}{ iUsted se ha caído? } \\
\cline { 2 - 6 } & & \multicolumn{1}{c}{ Si } & \multicolumn{1}{c}{ No } & $\%$ \\
\cline { 2 - 6 } & $60-65$ años & 6 & 12,8 & 2 & 11,8 \\
& $66-70$ años & 12 & 25,5 & 4 & 23,5 \\
Edad & $71-75$ años & 9 & 19,1 & 2 & 11,8 \\
& $76-80$ años & 8 & 17,0 & 4 & 23,5 \\
& $81-85$ años & 7 & 14,9 & 3 & 17,6 \\
\hline Total & 86 y más años & 5 & 10,6 & 2 & 11,8 \\
\hline \multirow{2}{*}{ Sexo } & & 47 & 100,0 & 17 & 100,0 \\
\hline Total & Mujer & 30 & 63,8 & 10 & 58,8 \\
\hline \multirow{2}{*}{ Miedo de sufrir una caída } & Si & 17 & 36,2 & 7 & 41,2 \\
\hline Total & No & 47 & 100,0 & 17 & 100,0 \\
\hline
\end{tabular}


ferida por el AM, se encontró que en mayor porcentaje se debió a tropiezo $53,2 \%(n=25)$, seguido de resbalón 19,1\% ( $\mathrm{n}=9)$, mareo $19,1 \%(n=9)$, y otra causa $8,5 \%(n=4)$, entre las que se reportaron caída de la cama y caída de la silla.

\section{DISCUSIÓN Y CONCLUSIÓN}

La investigación responde al objetivo de evaluar el uso del bastón y la prevalencia de caídas en el AM, además reafirma lo encontrado en otros estudios sobre caídas y aporta información sobre un tema que en el contexto nacional era desconocido. La muestra estuvo integrada en mayor número por mujeres, lo que es una tendencia en esta población, pues a nivel nacional concuerda con datos de caracterización sociodemográfica (23), cifras sobre requerimiento de ayudas técnicas (18), y otros resultados de estudios en AM $(15,16$, 24); en el plano internacional concuerda con estudios sobre caídas $(7,13)$ y uso de ayudas técnicas en AM $(1,25)$. En cuanto a la edad, el rango que concentró la mayor cantidad de AM fue el de 70 años y más, que concuerda con datos de caracterización de esta población (23). La media de edad de quienes presentaron caídas fue de 76 años, cifra que se asemeja a lo reportado en Chile (15) y a nivel internacional (20). En cuanto a la educación, el porcentaje de AM analfabetos es cercano a lo reportado en Chile y respecto a vivir solos, el dato también concuerda con los antecedentes a nivel nacional (26). Sobre la prevalencia de enfermedades crónicas, los resultados se presentan en el mismo orden que lo reportado en Chile (26), pero llama la atención que las cifras sean muy superiores, lo que quizá se deba a las características culturales y hábitos de alimentación de la población estudiada. Debemos considerar también, que es común que los AM presenten enfermedades crónicas que tienen a menudo un comportamiento incierto, donde el consumo de medicamen- tos se hace obligatorio y no exento de algunas complicaciones, como pueden ser las caídas. En este estudio, la media de consumo diario de medicamentos fue superior a lo reportado a nivel nacional (26), que se explicaría por las enfermedades crónicas y la posible automedicación en que incurren los AM; y en cuanto a las caídas por número de medicamentos, se asemeja a lo reportado a nivel internacional (27).

Destaca una alta prevalencia de caídas, al considerar un evento en los últimos seis meses, lo que fue muy superior a lo encontrado en un estudio nacional (17) e internacional (20) en AM de la comunidad. Llama la atención que la prevalencia se mantuvo alta al considerar dos o más caídas en seis meses, incluso por sobre otros resultados de estudios realizados en Chile que consideraron las caídas en el mismo tiempo (15) y en un año (17). Algo a considerar es que quizá la alta prevalencia de caídas se deba al bajo uso del bastón sumado al deterioro de la marcha y equilibrio; por su parte la diferencia de resultados con otros estudios puede atribuirse a que los otros estudios no consideraron una muestra de AM usuarios de bastón, por incorporar diferentes grupos de edad, presencia de enfermedades crónicas, nivel de actividad y barreras del ambiente, entre otros.

En cuanto al lugar de la caída, en general, se presentaron en mayor porcentaje fuera de la vivienda, lo que concuerda con otro reporte nacional (15). En lo específico, los AM se caen más en un lugar conocido, es decir, en el interior de la vivienda y el patio, que puede ser por el exceso de confianza y seguridad al percibir estar adaptados de cierta manera al entorno en función de sus competencias, llevándolos a minimizar los riesgos al momento de caminar y quizá a disminuir la atención sobre el entorno al momento de movilizarse. Por ello, se requiere realizar otras investigaciones, considerando el nivel de actividad de los AM en los diferentes espacios en que se movilizan, como también sus competencias, relacionadas con: salud cognitiva, síntomas 
depresivos, marcha y equilibrio; el ambiente físico, para conocer su entorno en función de la accesibilidad y uso de los recursos de la vivienda, y también sobre el ambiente social; pues se sabe que el AM tiene preferencia por permanecer más en la casa en desmedro de realizar otras actividades fuera de ella (2), lo que puede interferir en que lleve a cabo acciones de protección para su salud.

De quienes presentaron una o más caídas, un alto porcentaje vivía con una o más personas, que concuerda con lo reportado en Chile (15). Esto se puede deber a que al convivir con otras personas exista un mayor número de barreras en el ambiente, como también, a que sobreprotejan al AM llevándolo a un estado de baja competencia, que afectaría su relación con el entorno y consecuente riesgo de caer. Con respecto a la causa de la caída, referida por el AM, se encontró que en un mayor porcentaje se debió a tropiezo, misma causa reportada en otro estudio (15), que puede ser atribuible a problemas de la persona, como: alteración de la marcha, falta de atención, problemas visuales; o del ambiente físico, tales como: diferencias de nivel, mala iluminación y objetos en el piso; que por lo general se conjugan a la hora de presentar una caída. A su vez, la media de edad de quienes presentaron caídas se asemeja, en parte, con otros estudios sobre caídas en AM de la comunidad $(15,20)$, y en cuanto al sexo, las mujeres presentan mayor prevalencia de caídas, lo que ha sido ampliamente documentado $(7,15-17,20)$.

En otro orden de ideas, el tiempo de uso del bastón de los AM que recibieron la ayuda técnica en el Centro de Salud Familiar fue principalmente superior a un año, lo que concuerda con otro estudio (1); sobre ello es necesario destacar que el total de los AM recordaron el tiempo de uso, lo que difiere con un estudio a nivel internacional (25), que en ese caso puede atribuirse a deterioro cognitivo. Respecto al uso del bastón y caídas, existen datos contradictorios en lo referido por los AM, esto porque en su mayoría se sen- tían más seguros al caminar con el bastón, lo que se encuentra documentado (28); y en gran porcentaje reconocieron saber por qué debían utilizar el bastón, pero esos antecedentes se contraponen con el porcentaje que manifestó que siempre utilizaban la ayuda técnica, sea dentro o fuera de la casa, que fue inferior al 50\%. Cabe señalar que este último dato es muy similar al porcentaje de AM que manifestó que le agradaba utilizar el bastón, lo que explicaría en parte su bajo uso.

La no utilización del bastón se puede atribuir a la elección de los AM por el apoyo en otra persona para caminar, en desmedro del bastón, el entorno de la vivienda, las actividades del hogar y la percepción de su limitación funcional. Es decir, el no utilizar el bastón en la casa puede ser porque el AM se siente más seguro al estar en un lugar conocido y de alguna manera se ha adaptado a las barreras del ambiente, como también, que las condiciones de la vivienda no le permitan un fácil desplazamiento al caminar con el bastón, como pueden ser pasillos estrechos o marcadas diferencias de nivel. En ese sentido, se ha reportado que los AM con limitación funcional y uso de ayudas técnicas tienen problemas frecuentes de accesibilidad en sus viviendas (29). Otra explicación puede ser porque el AM debe realizar tareas en el hogar que serían incompatibles con utilizar el bastón, o por la percepción respecto a su grado de limitación funcional y consecuente adaptación, que lo llevaría a determinar que no es necesario utilizar el bastón en forma permanente. Esto abre un área de oportunidad para el desarrollo de nuevos estudios sobre ayudas técnicas y caídas, abordando otras variables, como pueden ser actividades de la vida diaria, salud cognitiva y barreras de la vivienda.

De acuerdo con los resultados obtenidos, da la impresión que el objetivo por el que se indican ayudas técnicas cuando un AM presenta limitación funcional para desplazarse y/o dificultad para realizar actividades de la vida diaria (4), no se está cumpliendo, por lo menos en este grupo, pues si el bastón no se 
utiliza se mantendrá la condición del AM y las dificultades al interactuar con el ambiente no disminuirán y por consiguiente se conservará el riesgo de sufrir accidentes que, en su conjunto, pueden conducir a un deterioro progresivo de su condición de salud. Asimismo, el dato sobre uso del bastón es relevante si consideramos las caídas, pues el $61 \%$ de quienes presentaron el evento no estaba usando el bastón al momento de caer. Sobre este resultado se necesita investigación adicional que permita comprender de mejor manera el uso del bastón, la frecuencia de caídas y el riesgo de caer en esta población, pues el otro porcentaje, a pesar de utilizar el bastón igual presentó caídas. Sería interesante evaluar el correcto uso de la ayuda técnica en diferentes aspectos, por ejemplo, la altura, en qué mano lo utilizan y la postura corporal; pues se han reportado errores en cuanto al cumplimento de la indicación (30), lo que puede conducir a no lograr el objetivo por el cual se indicó y a incrementar el riesgo de caer o el número de caídas. También sería beneficioso estudiar las indicaciones que se dan a los AM y el reforzamiento que se debe realizar, pues llama la atención que no todos manifestaron haber recibido educación de cómo utilizar el bastón por parte de un profesional. Ante ello queda también la incertidumbre si el AM recibió la instrucción necesaria respecto a la utilización de la ayuda técnica considerando diferentes escenarios, por ejemplo, su uso en lugares con diferencia de nivel. En ese sentido se ha recomendado educar sobre uso del bastón abordando distintos escenarios por donde el AM camina, como pueden ser las escaleras, pues en ese caso el bastón debería ser colocado en posición diferente a la utilizada cuando camina por otra superficie (31), de esta manera se busca disminuir el riesgo de accidentes.

Los resultados de este estudio sumado a la evidencia sobre el tema sustentan la relevancia de la educación sobre el uso de ayudas en AM, la que debe involucrar a toda la población, a la familia y equipo de salud, apun- tando a que se valoren de forma positiva las ayudas técnicas en función de sus beneficios y sin enmarcarlas en un grupo de edad. La educación debe estar ajustada al nivel de estudio, condiciones auditivas, visuales y cognitivas, utilizando herramientas metodológicas y materiales que permitan que la entrega de información sea efectiva.

La información sobre el uso del bastón también conduce a analizar las características de la ayuda técnica que se entrega en los Centros de Salud, considerando aspectos como forma, color, tipo de material y ajuste en altura, pues al parecer son aspectos claves que estarían afectando el uso por parte de la población de AM, a lo que se agrega el componente social. Se debe considerar también que los AM han convivido, en cierta medida, con un entorno tecnológico, en virtud de ello las ayudas técnicas como el bastón; deben ser desarrolladas en función de los nuevos intereses de las personas.

Ahora bien, desde que los AM tienen el bastón, más de la mitad refirió caminar menos, lo que se puede explicar, en parte, por el estigma social y el agrado de uso. En ese sentido los profesionales de la salud deben reforzar la importancia de la utilización del bastón como también sobre los beneficios de caminar y de mantener y/o incrementar el nivel de actividad física, pues en quienes están con limitación funcional existe la evidencia que caminar en forma regular puede conferir protección a corto plazo de la pérdida adicional de la movilidad (32).

Si se considera que entre los propósitos de la indicación del bastón están proporcionar apoyo, optimizar la función de la persona y la independencia (31), conviene destacar que en el grupo estudiado alguno de esos aspectos no se están cumpliendo. Esto quizá se deba a que un alto porcentaje de los participantes expresó tener miedo de sufrir una caída, cifra que aumenta cuando solo se evalúa a aquellos que presentaron una o más caídas; esto afectaría el nivel de actividad del AM y su independencia. Los resultados evidencian 
que el miedo a caer está por sobre lo reportado a nivel internacional de los AM que utilizan bastón y que tienen antecedente de caída (1); y se sabe que el miedo a caer es un factor de riesgo para presentar caídas (8). Este antecedente sin duda requiere mayor profundización en futuras investigaciones, porque, por un lado, los AM manifestaron sentirse más seguros al caminar con bastón, pero también reportan en alto porcentaje miedo a caer, el que quizá sea responsable del poco uso del bastón, del nivel de actividad que realizan y de las caídas.

Considerando el incremento de la población de AM, fenómeno global que se espera persista, se sugiere continuar realizando estudios abordando la interacción ambiental y social de los AM, de esta forma se obtiene información relevante para realizar mejoras en los programas e intervenciones efectivas para proporcionar una mejor calidad de vida a esta población. En ese sentido los profesionales de enfermería tienen un rol relevante respecto a las ayudas técnicas y caídas, al abordar actividades de promoción y prevención, pues están en contacto permanente con el AM, su familia y comunidad, considerando los controles de salud y visitas domiciliarias.

El cambio demográfico también propone desafíos para las universidades, como insertar la temática de las caídas en los programas de asignatura de las carreras de la salud, abordando los diferentes grupos de edad, factores de riesgo de la persona y del ambiente, pues hasta ahora tienden a enfocarse principalmente en sus consecuencias.

Como limitaciones de este estudio cabe mencionar la selección y tamaño de la muestra. Con todo, la principal conclusión del estudio es que un alto porcentaje de AM no está cumpliendo con el uso del bastón, por lo tanto el objetivo de su entrega no se está logrando; evidencia indiscutible y hasta ahora desconocida en Chile. Asimismo, de quienes presentaron caídas, un alto porcentaje no estaba usando el bastón al momento de caer. En consecuencia, se debe incluir la evaluación y educación sobre uso de bastón y caídas como parte del control de salud del AM.

\section{REFERENCIAS}

1. Gell NM, Wallace RB, LaCroix AZ, Mroz TM, Patel KV. Mobility device use in older adults and incidence of falls and worry about falling: findings from the 20112012 National Health and Aging Trends Study. J Am Geriatr Soc. 2015; 63(5): 85359.

2. Servicio Nacional del Adulto Mayor, Ministerio de Desarrollo Social. Boreal, Investigación y Consultoría Ltda. Estudio de recopilación, sistematización y descripción de información estadística sobre vejez y envejecimiento en Chile. Informe Final [Internet]. Santiago, Chile: Ministerio de Desarrollo Social. Boreal, Investigación y Consultoría Ltda; 2011 [citado 13 mayo 2016]. 125 p. Disponible en: http:// www.senama.gob.cl/storage/docs/Informe-final-recopilacion-estadistica-la-realidad-de-los-mayores-en-Chile-2012.pdf

3. Ministerio de Salud (MINSAL). Guía Clínica Endoprótesis Total de Cadera en personas de 65 años y más con Artrosis de Cadera con Limitación Funcional Severa [Internet]. Santiago, Chile: MINSAL; 2010. [citado 13 mayo 2016]. Disponible en: http://www.bibliotecaminsal. cl/wp/wp-content/uploads/2016/04/Endopr\%C3\%B3tesis-para-Artr\%C3\%B3sis-de-Cadera-65-a\%C3\%B1os-y-m\%C3\%A1s.pdf

4. Ministerio de Salud (MINSAL). Guía Clínica Órtesis (o ayudas técnicas) para personas de 65 años y más [Internet]. Santiago, Chile: MINSAL; 2010. [citado 13 mayo 2016]. Disponible en: http://www. bibliotecaminsal.cl/wp/wp-content/ uploads/2016/04/rtesis.pdf

5. Ministerio de Salud (MINSAL), Superintendencia de Salud, Orientación en 
Salud, Problema de Salud Auge No 36, Órtesis (o ayudas técnicas) para personas de 65 años y más. [Internet]. [citado 13 mayo 2016]. Disponible en: http://www. supersalud.gob.cl/difusion/665/w3-article-610.html

6. Löfquist C, Nygren C, Brandt Å, Iwarsson $S$. Very old Swedish women's experiences of mobility devices in everyday occupation: a longitudinal case study. Scand J Occup Ther. 2009; 16(3): 181-92.

7. Iwarsson S, Horstmann V, Carlsson G, Oswald F, Wahl, HW. Person-environment fit predicts falls in older adults better than the consideration of environmental hazards only. Clin Rehabil. 2009; 23(6): 558-67.

8. Kwan MM, Close JC, Wong AK, Lord SR. Falls incidence, risk factors, and consequences in Chinese older people: A systematic review. J Am Geriatr Soc. 2011; 59(3): 536-43.

9. Chang N, Yang N, Chou P. Incidence, risk factors and consequences of falling injuries among the community-dwelling elderly in Shihpai, Taiwan. Aging Clin Exp Res. 2010; 22(1): 70-7.

10. González JI, Alarcón T. Grandes Síndromes Geriátricos. Concepto y Prevención de los más importantes. Medicine. 2003; 8(108): 5778-85.

11. Oudshoorn C, Hartholt KA, Zillikens MC, Panneman MJ, Van der Velde N, Colin EM, et al. Emergency department visits due to vertebral fractures in the Netherlands, 1986-2008: Steep increase in the oldest old, strong association with falls. Injury. 2012; 43(4): 458-61.

12. Craig, J, Murray A, Mitchell S, Clark S, Saunders L, Burleigh L. The high cost to health and social care of managing falls in older adults living in the community in Scotland. Scott Med J. 2013; 58(4): 198-03.

13. Hartholt KA, Polinder S, Van der Cammen TJ, Panneman MJ, Van der Velde $\mathrm{N}$, Van Lieshout EM, et al. Costs of falls in an ageing population: A nationwide study from the Netherlands (2007-2009). Injury. 2012; 43(7): 1199-203.

14. Siracuse JJ, Odell DD, Gondek SP, Odom SR, Kasper EM, Hauser CJ, et al. Health care and socioeconomic impact of falls in the elderly. Am J Surg. 2012; 203(3): 33538.

15. González G, Marín PP, Pereira G. Características de las caídas en el adulto mayor que vive en la comunidad. Rev Med Chil. 2001; 129(9): 1021-30.

16. Gac H, Marín PP, Castro S, Hoyl T, Valenzuela E. Caídas en adultos mayores institucionalizados: Descripción y evaluación geriátrica. Rev Med Chil. 2003; 131(8): 887-94.

17. Reyes-Ortiz CA, Al Snih S, Markides KS. Falls among elderly persons in Latin America and the Caribbean and among elderly Mexican-Americans. Rev Panam Salud Publica. 2005; 17(5-6): 362-69.

18. Tamayo M, Aleitte F. Cumplimiento de garantía de oportunidad GES en ayudas técnicas en una comuna de la Región Metropolitana. Rev Chil Salud Pública. 2011; 15(3): 146-54.

19. Betz ME, Kelly SP, Fisher J. Death Certificate Inaccuracy and Underreporting of Injury in Elderly People. J Am Geriatr Soc. 2008; 56(12): 2267-72.

20. Leiva-Caro JA, Salazar-González BC, Gallegos-Cabriales EC, Gómez-Meza MV, Hunter KF. Connection between competence, usability, environment and risk of falls in elderly adults. Rev Lat Am Enfermagem. 2015; 23(6): 1139-48.

21. Ministerio de Salud, Subsecretaría de Salud Pública. Ley 20120, sobre la investigación científica en el ser humano, su genoma, y prohíbe la clonación humana [Internet]. Única versión, 22 de septiembre de 2006. Biblioteca del Congreso Nacional de Chile. [citado 17 mayo 2016]. Disponible en: http://www.leychile.cl/ Navegar?idNorma $=253478$

22. Ministerio Secretaría General de la Presi- 
dencia. Ley 19.628, sobre protección de la vida privada [Internet]. Última versión, 17 de febrero de 2012. Biblioteca del Congreso Nacional de Chile. [citado 17 mayo 2016]. Disponible en: http://www.leychile.cl/Navegar?idNorma=141599

23. Ministerio de Desarrollo Social. Encuesta de Caracterización Socioeconómica Nacional Adultos Mayores Síntesis de Resultados [Internet]. Santiago: Ministerio de Desarrollo Social; 2013 [citado 18 mayo 2016]. 63 p. Disponible en: http://observatorio.ministeriodesarrollosocial.gob. cl/documentos/Casen2013_Adultos_mayores_13mar15_publicacion.pdf

24. Ferrada L, Zavala M. Bienestar psicológico: adultos mayores activos a través del voluntariado. Cien. enferm. 2014; XX(1): 123-30.

25. Härdi I, Bridenbaugh SA, Gschwind YJ, Kressig RW. The effect of three different types of walking aids on spatio-temporal gait parameters in community-dwelling older adults. Aging Clin Exp Res. 2014; 26(2): 221-28.

26. Servicio Nacional del Adulto Mayor. Chile y sus mayores 2013. Resultados Tercera Encuesta Nacional Calidad de Vida en la Vejez 2013 [Internet]. Santiago: Vicerrectoría de Comunicaciones y Educación Continua, Pontificia Universidad Católica de Chile; 2013. [citado 13 mayo 2016]. 103 p. Disponible en: http://www.senama.cl/filesapp/Chile\%20y\%20sus $\% 20$ mayores $\% 202013, \% 20$ Encuesta $\% 20$ de\%20Calidad\%20de\%20Vida.pdf

27. Varas-Fabra F, Castro E, Pérula de Torres LA, Fernández MJ, Ruiz R, Enciso I. Caídas en ancianos de la comunidad: prevalencia, consecuencias y factores asociados. Atención Primaria. 2006; 38(8): 450-55.

28. Aminzadeh F, Edwards N. Factors associated with cane use among community dwelling older adults. Public Health Nurs. 2000; 17(6): 474-83.

29. Lien LL, Steggell CD, Iwarsson S. Adaptive strategies and person-environment fit among functionally limited older adults aging in place: a mixed methods approach. Int J Environ Res Public Health. 2015; 12(9): 11954-74.

30. Liu HH, Eaves J, Wang W, Womack J, Bullock P. Assessment of canes used by older adults in senior living communities. Arch Gerontol Geriatr. 2011; 52(3): 299-03.

31. Hsue BJ, Su FC. The effect of cane use method on center of mass displacement during stair ascent. Gait Posture. 2010; 32(4): 530-35.

32. Simonsick EM, Guralnik JM, Volpato S, Balfour J, Fried LP. Just Get Out the Door! Importance of Walking Outside the Home for Maintaining Mobility: Findings from the Women's Health and Aging Study. J Am Geriatr Soc. 2005; 53(2): 198-203. 